\title{
Paraneoplastic cerebellar degeneration
}

\author{
$A$ clinical and CT study \\ Harry S Greenberg \\ Department of Neurology, University of Michigan Medical School, USA
}

Keywords: paraneoplastic cerebellar degeneration, CT scanning

\section{Summary}

Six patients developed a pancerebellar syndrome with symptoms preceding the diagnosis of neoplasia in five (median - 4 months) and following in one ( 2 years). In all patients, the initial cranial computed tomographic (CT) scans were normal. Five patients had repeat CTs and of these three were abnormal; cerebellar atrophy appearing 7 to 25 months following the initial CT. Median follow-up was 31 months (range 12-84 months) without evidence of CNS metastatic disease. In five of six patients the neurologic impairment did not progress. One patient's neurologic signs improved markedly with mantle radiation therapy of her Hodgkin's disease. An initially negative CT does not preclude the diagnosis of remote effect cerebellar atrophy. Paraneoplastic cerebellar degeneration is a self-limited nonprogressive process in the majority of patients.

\section{Introduction}

Neurologic dysfunction in patients suffering from cancer can be caused by either metastases of the primary tumor to the nervous system or through non-metastatic tumor effects on the nervous system. Neurologic remote effects of cancer are disorders, cause unknown, occurring with greater frequency in patients with cancer. The remote effect cerebellar syndrome is a rare entity where symptoms often precede the diagnosis of malignancy. In adults with an unexplained cerebellar syndrome a careful search for primary malignancy with good follow-up is necessary.

Presented in part at the 35 th Annual Meeting of the American Academy of Neurology, San Diego, CA, April 28-30, 1983.

\section{Methods}

This report reviews six cases of remote effect cerebellar degeneration diagnosed during a fiveyear period at four institutions. A seventh case was excluded because the history and clinical findings supported a diagnosis of alcoholic cerebellar degeneration. The sixth case was published previously and is included with permission of the authors (1). Two representative cases are reported.

\section{Case 1}

A 57-year-old female had the onset of diplopia and blurry vision on June 6, 1979. Nine days later, slurred speech occurred and was followed by vertigo, nausea, and vomiting. Nystagmus was observed on exam and initial work-up including skull X-rays and CT was negative. In August 1979 she was examined at an outside hospital, found to have a 
'stiff-legged', ataxic gait and rotary nystagmus on horizontal gaze bilaterally. There was vertical nystagmus in the direction of gaze. Deep tendon reflexes were brisk with a $2+$ jaw jerk and flexor plantar responses. CT with posterior fossa views (Fig. 1 - top) was negative. SMA 12, CBC, TSH, T4, ESR, ANA, rheumatoid factor and CPK were negative.

In October 1979 she was admitted to another hospital with incomplete partial bowel obstruction and an exploratory laparotomy and left salpingooophorectomy showed a poorly differentiated ovarian serous adenocarcinoma. In December 1979 she underwent right salpingo-oophorectomy, hysterectomy, and omentectomy. Following operation, cerebellar symptoms were worse. She was begun on alkeran $4 \mathrm{mg}$ orally three times a day for five days monthly in March 1980. Between January 1980 and August 1981 no change was noted in coordination, speech or strength. In August 1981 general physical exam revealed a moderately obese female and was otherwise unremarkable. Cranial nerve exam showed horizontal and vertical nystagmus in the direction of gaze. Gait was ataxic, requiring assistance with support to bear weight.
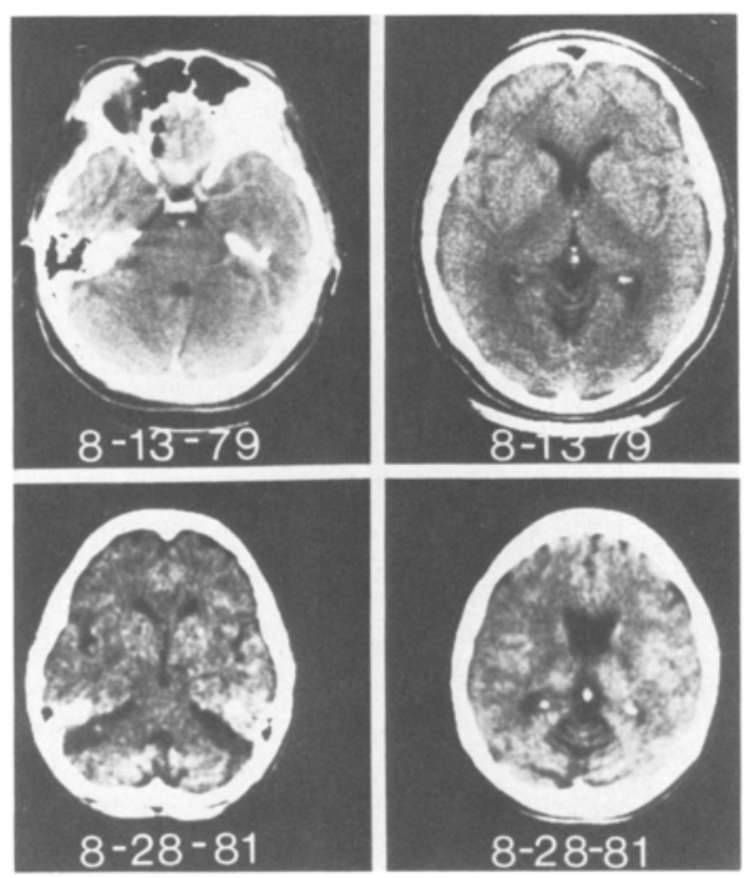

Fig. 1. (Top): Normal CT at onset of illness. (Bottom): Two years later with patient stabilization $C T$ shows marked lateral cerebellar hemisphere and vermian atrophy.
Finger-nose-finger and heel-knee-shin testing showed symmetric dysmetria. Tone was increased in all four extremities with normal strength. Reflexes were unchanged from the initial examination. Sensory exam was normal. Laboratory data: Hgb 12.4, Hct 36.8, WBC 5,100, PLT 440,000, TP 8.7, albumin 3.7, alkaline phosphatase 143 , with the rest of the SMA normal. B12 and folate were normal. Repeat CT of the posterior fossa showed marked cerebellar atrophy (Fig. 1 - bottom). Abdominal CT showed massive periaortic tumor. Adriamycin and cisplatinum chemotherapy were begun. The patient was alive on August 1, 1982 without change in her neurologic deficit from March 1980.

\section{Case 2}

A 20-year-old healthy orthodox seminary student developed headache, neck pain, drowsiness and vertigo accompanied by shaking chills, diaphoresis, nausea, vomiting and a temperature to $102{ }^{\circ} \mathrm{F}$. She was admitted to an outside hospital and cervical lymph node hyperplasia was noted. On neurologic exam she was found to have a labile affect and generalized ataxia. A cervical lymph node biopsy and lumbar puncture were performed and she was transferred to the University of Michigan Hospital on September 8, 1980. Lumbar puncture protein was 56 , glucose $53,2 \mathrm{RBC}, 2 \mathrm{WBC}$ ( $100 \%$ lymphocytes), negative cultures and cytology.

On admission her blood pressure was $120 / 80$, pulse 80 , respiration 16 , and temperature $98.6^{\circ} \mathrm{F}$. Her general physical exam was normal except for a surgical scar in the right anterior neck region and guarding in the left upper quadrant of the abdomen on deep palpation.

On mental status exam she was oriented and had slurred speech. Her affect was impulsive, childlike and inappropriate. She denied gait difficulty. She remembered 3 out of 3 objects at five minutes and one hour. Serial sevens were performed well and proverb interpretation was abstract. Cranial nerves were normal except for gaze induced horizontal nystagmus. Her supported gait was wide-based and ataxic. Severe dysmetria and dystaxia was present in all four extremities. Her strength was normal. Reflexes were $2+$ bilaterally with flexor plantar responses. Sensory exam was normal. 
Initial laboratory data: Hct $38.6, \mathrm{Hgb} 11.9$, platelet 621,000 , WBC 24,000 with a differential of $62 \%$ segs, $27 \%$ bands, $1 \%$ myelocytes, $9 \%$ lymphocytes and $1 \%$ eosinophils. Chemistries were: sodium 141 , potassium 4.3 , chloride $98, \mathrm{CO}_{2} 23, \mathrm{BUN} 14$, creatinine 0.8 , glucose 101 , calcium 10.3, phosphorous 4.2 , protein 8.5 , albumin 4.0 , total bilirubin 0.3 , SGOT 35, SGPT 39, LDH 770, alkaline phosphatase 74, and magnesium 2.0. Chest X-ray showed moderate scoliosis and right hilar mass. Lymph node biopsy was reported as nodular sclerosing Hodgkin's disease. EEG on September 11, 1980 showed diffuse slowing of the background to 7 $1 / 2 \mathrm{~Hz}$. No laboratory tests were obtained between September 12-16, 1980 because of religious reasons. CT with and without contrast on September 17, 1980 was normal (Fig. 2A). Repeat lumbar puncture revealed a protein of 36 , glucose 52 , 13 R BC, 13 WBC ( $98 \%$ lymphocytes and $2 \%$ histio-

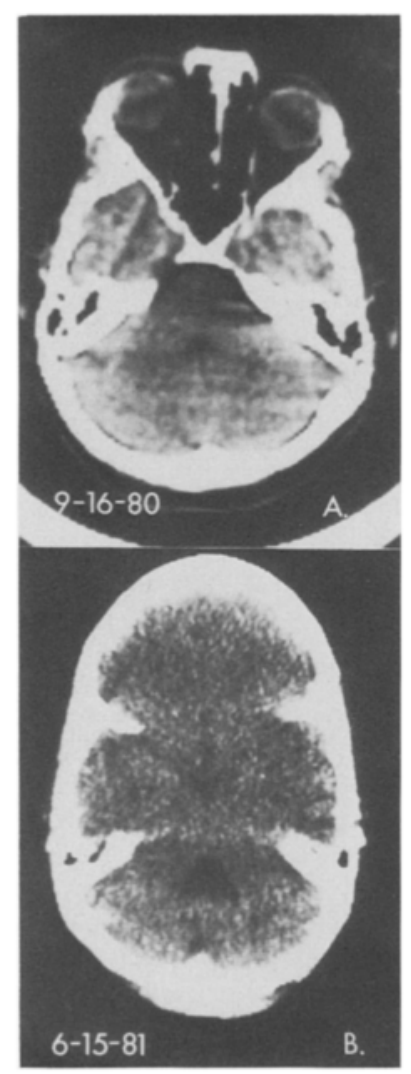

Fig. 2. (A) CT shows normal cerebellum and fourth ventricle when patient has maximal neurologic deficit. (B) Nine months later CT shows marked enlargement of fourth ventricle at time when patient dramatically clinically improved. cytes). The IgG/albumin ratio was $21 \%$ with negative VDRL, cultures and cytology. There were no oligoclonal bands. Visual and brainstem auditory evoked responses were normal. Bone marrow biopsy and abdominal CT were normal. Gallium scan showed increased uptake in the right supraclavicular region, liver and spleen. Staging laparotomy on September 29, 1980 was negative for disease in the abdomen. The patient was treated with mantle radiotherapy. Her mental status progressively became normal during hospitalization. Eight months following discharge her only neurologic abnormality was a minimally wide-based stance and mild finger-nose-finger and heel-knee-shin dysmetria bilaterally. There was no evidence of active Hodgkin's disease. CT on June 15, 1981 showed marked enlargement of the fourth ventricle (Fig. 2B).

\section{Results}

Six cases of remote effect cerebellar degeneration are reported. In five of the six cases $(83 \%)$ the cerebellar symptoms preceded the diagnosis of neoplasia by a median of four months (range 0-56 months). Gait difficulty was the initial symptom in four, diplopia and vertigo in one each ( $\mathrm{Ta}-$ ble 1). At neurologic exam at the time of diagnosis all six were dysarthric with gait ataxia and limb dysmetria. Four patients had horizontal nystagmus and two of these vertical nystagmus.

Seven primary tumors were diagnosed in six patients. Two patients had nodular sclerosing Hogdkin's disease, and one patient each had acute myelomonocytic leukemia, ovarian carcinoma, lung carcinoma, with the sixth having both colon and uterine carcinoma. The four patients with Hodgkin's disease or gynecologic malignancy had pure cerebellar syndromes except for a transiently inappropriate affect in one (Table 1). In addition to their cerebellar syndrome, two patients had neuropathy, one with myasthenic syndrome and the other with bilateral extensor plantar responses. These two patients had an increased cerebrospinal fluid protein (Table 1). In all six patients the initial CTs were normal. Repeat CTs were obtained in five patients and were abnormal in three showing cerebellar atrophy, 7 to 25 months after the initial CT. Clinical follow-up was obtained in all six patients for a median of 31 months. Four patients are alive 


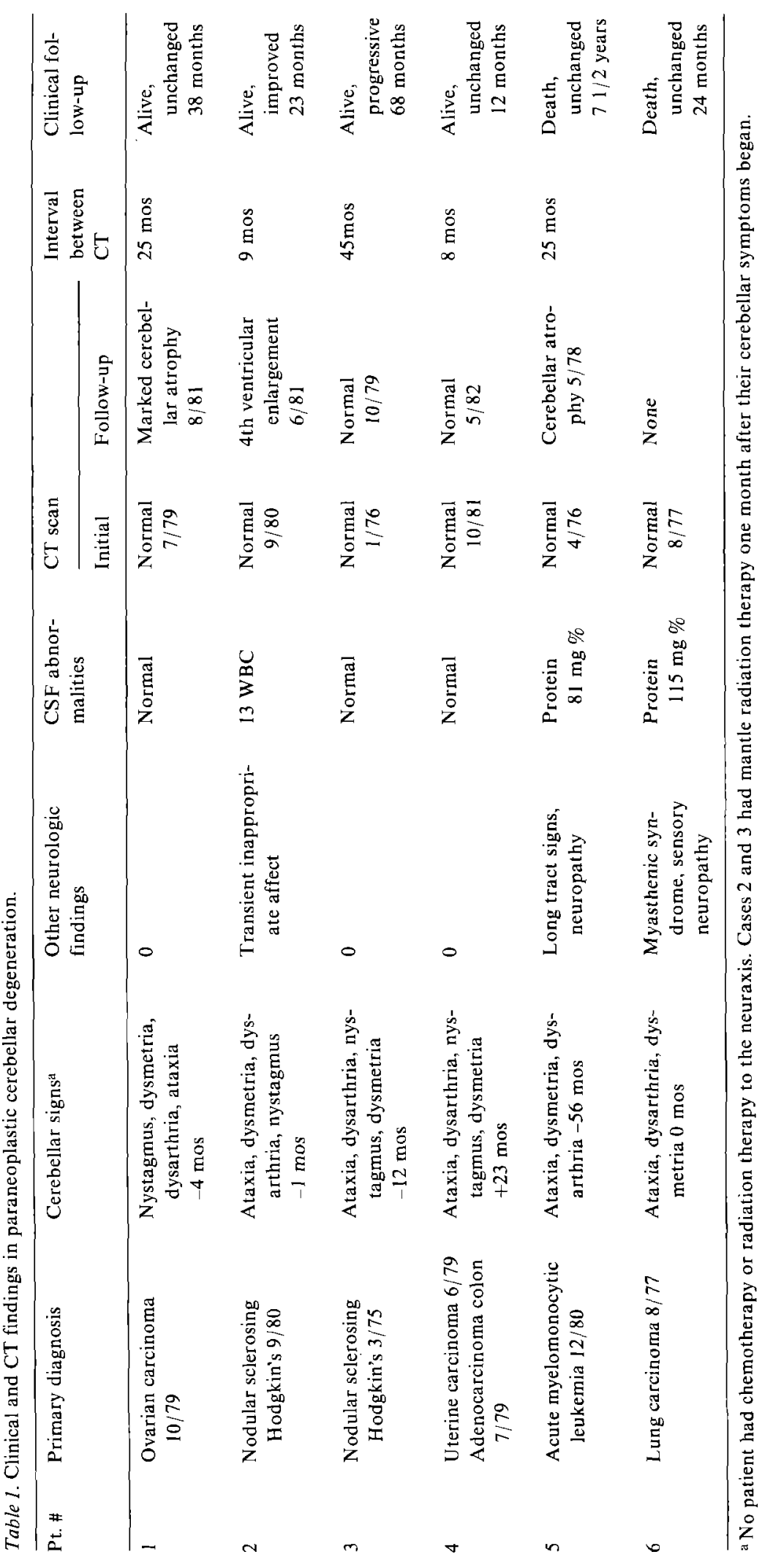


at a median of 30 months (range 12-68 months following diagnosis). No patient has developed evidence of CNS posterior fossa metastatic disease. One patient improved markedly following mantle radiation therapy and is now ambulatory with a minimally wide-based stance and only mild appendicular ataxia (Case 2). Four patients' neurologic impairment stabilized without further progression and one progressed slowly.

\section{Discussion}

The six patients reported here and the previous 60 cases in the literature describe a unique pancerebellar syndrome (1-12). The illness is characterized by the subacute onset of progressive, painless gait ataxia, dysmetria of the limbs, and dysarthria. In $83 \%$ of our cases the cerebellar symptoms and signs preceded the diagnosis of malignancy, which is slightly higher than the $63 \%$ reported in the literature by Brain et al. (2). In Henson and Urich's (3) experience approximately one-half of patients with late onset non-familial, cortical cerebellar degeneration will eventually develop a malignancy. None of our patients had antecedent cytotoxic chemotherapy or radiation directed to or overlapping the neuraxis (Table 1). The etiology of paraneoplastic cerebellar degeneration is unknown but in five patients, three secondary to ovarian carcinoma and one each secondary to Hodgkin's disease and breast carcinoma, circulating anti-Purkinje cell antibodies have been found, suggesting an immune-mediated disorder $(9,10,11,12)$. The antibody was an immunoglobulin $\mathrm{G}$ with cytoplasmic fluorescent clumping to Purkinje cell cytoplasm without clumping to other neuraxis sites or rat cerebellum(11). None of the patients with anti-Purkinje cell antibodies have had pathologic confirmation of diagnosis. One antibody positive and three antibody negative patients did not respond to plasmapheresis (11).

All patients had gross cerebellar abnormalities on initial exam (involving both the upper and lower limbs), although all CTs were initially normal. In the series of Brain et al. (2) 18 of 19 patients had upper and lower extremity cerebellar abnormalities. On repeat CT three of five cases developed abnormalities 7 to 25 months following initial CT with cerebellar atrophy and enlargement of the fourth ventricle. Henson and Urich (3) divided 43 pathologically proven cases into a pure cortical cerebellar degeneration [24] or with an inflammatory component in the meninges and perivascularly in the cerebellum [19]. In both types there is usually a severe or total loss of Purkinje cells. The granule cell layer may be involved but less than Purkinje cell layer, while the molecular layer is spared $(2,3)$. Degeneration of the long tracts of the spinal cord has been seen and may explain the extensor plantar responses in one patient (2). The delayed cerebellar atrophy seen on CT is consistent with autopsy pathology.

Prolonged follow-up in our six cases revealed that in five of the six patients this was a non-progressive syndrome. In four patients neurologic impairment stabilized and only one progressed during the course of their malignancy. One patient improved markedly after mantle radiation therapy of her Hodgkin's disease. Fourth ventricular enlargement appeared on her CT after dramatic clinical improvement. Three reports in the medical literature document significant improvement in the cerebellar dysfunction, one without treatment and two following treatment. In the first patient with colon carcinoma gait and appendicular ataxia remitted one month after onset before any treatment was given, while in the second improvement followed pneumonectomy for bronchogenic carcinoma (5, $6)$. In the second case the remission of symptoms began immediately after surgery and by seven days the patient was able to ambulate with a cane. At eleven months there was no signs of cerebellar disease (5). In the third patient with bronchogenic carcinoma and remote effect sensory polyneuropathy, cerebellar syndrome, and long tract signs, there was improvement from poor antigravity function being unable to keep his shin on the opposite heel to walking with a walker following $41 / 2$ months of therapy (7). Jaeckle et al. (11) found that the antibody positive patients $(2 / 6)$ had a steady progression of neurologic dysfunction whereas antibody negative patients had intervening periods of remission. However, of three antibody positive patients reported by Trotter (9) and Greenlee (12) only one had progressive disease over one year, the other two were stable. Although the paraneoplastic cerebellar syndrome caused major neurologic disability it was a self-limited non-progressive process in the majority of our patients. An 
initially negative CT scan does not preclude the diagnosis of remote effect cerebellar degeneration.

\section{References}

I. Zweifel TJ, Albers JW: Multiple neurologic paraneoplastic syndromes. Report of a case. Arch Neurol 37:315-316, 1980.

2. Brain $L$, Wilkinson $M$ : Subacute cerebellar degeneration associated with neoplasms. Brain 88:465-478, 1965.

3. Henson RA, Urich H: Cortical cerebellar degeneration. In: Cancer and the nervous system. Blackwell Scientific Publications, Oxford, England, 1982, pp 346-368.

4. Brazis PW, Biller J, Fine M, Palacios E, Pagano RJ: Cerebellar degeneration with Hodgkin's disease. Computed tomographic correlation and literature review. Arch Neurol 38:253-256, 1981.

5. Paone JF, Jeyasingham K: Remission of cerebellar dysfunction after pneumonectomy for bronchogenic carcinoma. New Eng J Med 302(3):156, 1980.
6. Auth TL, Chodo HP: Transient cerebellar syndrome from extracerebral carcinoma. Neurlogy 7:370-372, 1957.

7. Roberts HJV: A case of spinocerebellar degeneration associated with carcinoma of the prostate. Med J Austral 1:391-393, 1967.

8. Rewcastle NB: Subacute cerebellar degeneration with Hodgkin's disease. Arch Neurol 9:407-4I3, 1963.

9. Trotter JL, Hendin BA, Osterland CK: Cerebellar degeneration with Hodgkin's disease. An immunological study. Arch Neurol 33:660-661, 1976.

10. Greenlee JE: Is paraneoplastic cerebellar degeneration an immune-mediated condition? Detection of circulating antibodies to Purkinje cells in a patient with the disorder. Ann Neurol 12:103, 1982.

11. Jaeckle KA, Houghton AN, Nielsen SL, Posner JB: Demonstration of serum anti-Purkinje antibody in paraneoplastic cerebellar degeneration and preliminary antigenic determination. Ann Neurol 14:111, 1983.

12. Greenlee JE, Brashear HR: Antibodies to cerebellar Purkinje cells in patients with paraneoplastic cerebellar degeneration and ovarian carcinoma. Ann Neurol 14:609-613, 1983. 